\title{
Fitoplancton y zooplancton en el área marina protegida de Isla Gorgona, Colombia, y su relación con variables oceanográficas en estaciones lluviosa y seca
}

\author{
Alan Giraldo ${ }^{1 *}$, Bellineth Valencia ${ }^{1}$, Juan David Acevedo ${ }^{1} \&$ Marisol Rivera ${ }^{1}$ \\ 1. Departamento de Biología, Universidad del Valle, A.A. 25360 Cali, Colombia; alan.giraldo@correounivalle.edu.co, \\ oceanografía@univalle.edu.co
}

Recibido 18-X-2013. Corregido 20-XI-2013. Aceptado 19-XII-2013.

\begin{abstract}
Phytoplankton and zooplankton on the marine protected area of Gorgona Island and its relationship with hydrographic variables during the rainy and dry seasons. We studied the variation in abundance and biomass of plankton in the pelagic environment of Gorgona during October 2010 (rainy season) and March 2011 (dry season), and evaluated their correlation with hydrographic features of temperature, salinity, dissolved oxygen and transparency. Surface waters during both periods were warmer $\left(>26^{\circ} \mathrm{C}\right)$ and with low salinity values $(<31)$. At $30 \mathrm{~m}$ during October the waters were homogeneous, while during March the waters were colder $\left(<18^{\circ} \mathrm{C}\right)$, saltier $(>34)$ and presented low oxygen concentrations $(<3.0 \mathrm{ml} / 1) .61$ genera of phytoplankton and 30 zooplankton groups were identified, with greater values of phytoplankton genus and zooplankton abundance and biomass during March than in October. Bacteriastrum, Chaetoceros, Coscinodiscus, Guinardia, Rhizosolenia and Skeletonema were the phytoplankton genera most frequent in both sampling periods. Copepods were the dominant group of zooplankton, followed by chaetognaths and appendicularia in both periods. Zooplankton biomass was positively associated with surface temperature, salinity at $10 \mathrm{~m}$, and transparency of water column, and negatively associated with surface and 30m dissolved oxygen. Rev. Biol. Trop. 62 (Suppl. 1): 117-132. Epub 2014 February 01.
\end{abstract}

Key words: phytoplankton, zooplankton, temperature, salinity, hydrography, eastern tropical Pacific, Colombia, Isla Gorgona.

En el ambiente pelágico los organismos planctónicos son los principales productores primarios y secundarios, y por lo tanto, constituyen un enlace fundamental en la transferencia de energía en los ecosistemas marinos (Mann \& Lazier, 2006; Rubao, Edwards, Mackas, Runge \& Thomas, 2010). La importancia ecológica de esta comunidad radica, en que los patrones de variación temporal y espacial de su estructura, abundancia o biomasa pueden, en conjunto con otras variables físico-químicas de la columna de agua (e.g. temperatura, salinidad, oxígeno disuelto y nutrientes), modular la estructura de las comunidades bentónicas, así como la disponibilidad y viabilidad de recursos pesqueros comerciales de una región (Lalli \& Parsons, 1997; Miller, 2004; Brown et al., 2010).
Isla Gorgona (258'10" N - 78 $\left.11^{\circ} 05^{\prime \prime} \mathrm{W}\right)$ es el área insular más extensa $\left(13.3 \mathrm{~km}^{2}\right)$ en la zona sur del Pacífico colombiano. Esta isla, separada $30 \mathrm{~km}$ del continente fue declarada Parque Nacional Natural en 1985 debido a su alta diversidad biológica (Giraldo \& Valencia, 2012). El primer reporte de un estudio sistemático sobre la estructura y variación temporal de las comunidades planctónicas en isla Gorgona data de 1999, cuando Soto, Sánchez \& Fernández (2001) evaluaron la variación temporal en la composición y abundancia del fitoplancton y zooplancton, registrando las máximas abundancias para estos dos grupos durante marzo y diciembre. Posteriormente, Giraldo, Valencia \& Ramírez (2011) describieron la producción primaria y secundaria del ambiente pelágico 
de esta localidad junto con algunas variables físico-químicas de la columna de agua durante julio de 2006. Estudios más específicos para el zooplancton han identificado 35 especies de ictioplancton con una mayor abundancia en el sector sur de la isla (Escarria, Beltrán-León, Giraldo \& Zapata, 2007), y 46 especies de anfípodos hipéridos (Valencia \& Giraldo, 2012), con mayores valores de abundancia y diversidad durante el periodo de noviembre a marzo.

En isla Gorgona, la producción primaria y secundaria de la columna de agua presenta una variación estacional contrastante, estando modulada por las variaciones en el clima y la hidrografía, condiciones que responden a procesos que ocurren tanto a escala regional como local (Giraldo, Rodríguez-Rubio \& Zapata, 2008). A una escala regional, la variación estacional es modulada principalmente por el desplazamiento de la Zona de Convergencia Intertropical (ZCIT) y la consecuente intensificación del jet de viento de Panamá durante la época seca (diciembre-abril) (Eslava, 1993; Díaz, Pinzón, Perdomo, Barrios \& López-Victoria, 2001; Poveda \& Mesa, 2000; Amador, Alfaron, Lizano \& Magaña, 2006; Giraldo et al., 2008; Blanco, 2009), que genera el ingreso de aguas subsuperficiales frías $\left(<20^{\circ} \mathrm{C}\right)$, salinas $(>34)$ y ricas en nutrientes al Pacífico colombiano, (Forsbergh, 1969; Rodríguez- Rubio \& Stuardo, 2002; Fiedler \& Talley, 2006; D'Croz \& O'Dea, 2007). Estas aguas, advectadas desde la zona central de la cuenca del Océano Pacífico colombiano, conforman a lo largo de la zona nerítica la Corriente de Colombia (Rodríguez-Rubio, Schneider \& Abarca del Rio, 2003; Devis-Morales, Schneider, Montoya-Sánchez \& Rodríguez-Rubio, 2008), corriente que influencia las condiciones oceanográficas de la región costera, siendo reportado para isla Gorgona el ascenso de la termoclina $\left(7.5 \mathrm{~m}\right.$-isoterma de $\left.22^{\circ} \mathrm{C}\right)$, con la consecuente entrada de aguas frías y salinas entre febrero y abril (Díaz et al., 2001; Giraldo, 2008; Giraldo et al., 2008).

En contraste, durante la época de lluvias (mayo-noviembre) el jet de Panamá se debilita, y la circulación en el Pacífico colombiano está dominada por la formación de un giro anticiclónico en la zona central (Rodríguez-Rubio et al., 2003; Devis-Morales et al., 2008). Durante este periodo en isla Gorgona se registra la profundización en la termoclina (42.8-47.0m), así como aguas cálidas $\left(>25^{\circ} \mathrm{C}\right)$ y de baja salinidad (<34) (Díaz et al., 2001; Giraldo, 2008; Giraldo et al., 2008). Es también durante esta época cuando procesos locales como la desembocadura del río Patía-Sanquianga en el continente tienen su mayor influencia sobre la hidrografía de la isla, debido al aumento en los niveles de precipitación (Blanco, 2009), que contribuyen a su vez con el aporte de agua dulce y sedimentos en el ambiente pelágico del PNN Gorgona (Giraldo, 2008).

A pesar de la importancia del plancton en la transferencia de energía en los ecosistemas marinos, en isla Gorgona aún se carece de información básica sobre las variaciones estacionales en la composición, abundancia y producción (primaria y secundaria) de estos organismos en la columna de agua, así como su relación con la hidrografía de la región. Por esta razón, el objetivo del presente estudio fue evaluar la variación temporal de la estructura de la comunidad planctónica en isla Gorgona y explorar su relación con algunas variables hidrográficas.

\section{MATERIALES Y MÉTODOS}

Procedimiento de muestreo: Con el objetivo de describir la variación estacional en las condiciones hidrográficas y de la comunidad del plancton en la columna de agua de isla Gorgona, se realizó un muestreo durante la época de lluvias (24 al 26 de octubre de 2010) y otro durante la época seca (4 al 8 de marzo de 2011), siguiendo una malla de 24 estaciones alrededor de la isla (Fig. 1). En cada estación se determinó la transparencia del agua utilizando un disco Secchi, y se registró la distribución vertical de la temperatura y la salinidad hasta los $50 \mathrm{~m}$ (o cerca del fondo en estaciones someras) con un CTD Seabird-19. Adicionalmente, se recolectaron muestras de agua a tres profundidades estándares $(1 \mathrm{~m}, 10 \mathrm{~m}$ y $30 \mathrm{~m})$ utilizando una 


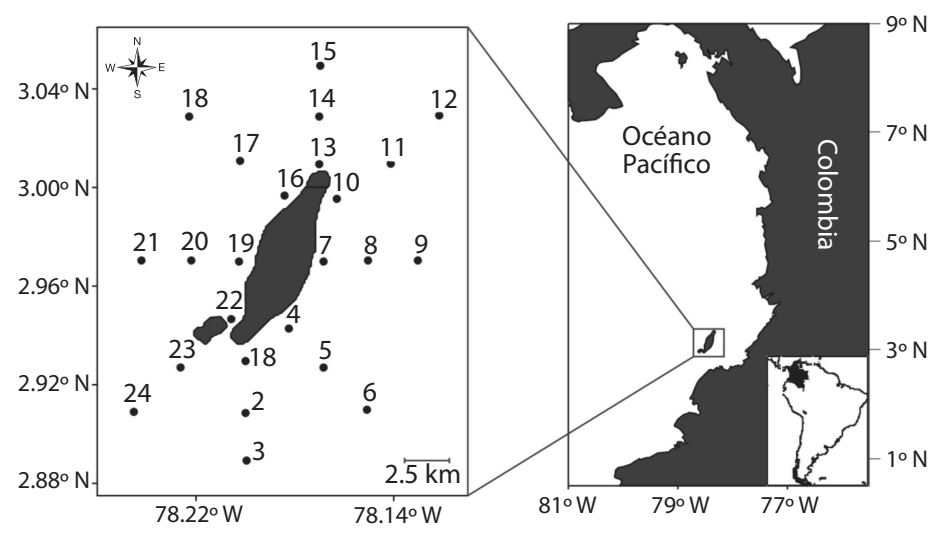

Fig. 1. Ubicación de las estaciones de muestreo en isla Gorgona (Pacífico colombiano).

Fig. 1. Location of sampling stations on Isla Gorgona (Colombian Pacific).

botella Niskin de 5L, con el fin de cuantificar la concentración del oxígeno disuelto utilizando una sonda multiparamétrica YSI85. Por limitaciones logísticas durante el muestreo de octubre de 2010, no fue posible obtener registros con el CTD en tres estaciones (E7, E20 y E21), y con el YSI85 en cinco estaciones (E1, E4, E7, E8 y E20).

Se realizaron arrastres superficiales durante 3 minutos utilizando una red cónica simple $(60 \mu \mathrm{m}$ ojo de malla, $30 \mathrm{~cm}$ diámetro) para recolectar fitoplancton en la zona de estudio. Por limitaciones técnicas con la red, durante el muestreo de octubre sólo se recolectaron muestras en 11 estaciones (E1, E4, E5, E6, E8, E9, E10, E11, E12, E23, E24). Las muestras de zooplancton se recolectaron mediante arrastres oblicuos desde $50 \mathrm{~m}$ de profundidad, o cerca del fondo en las estaciones más someras, hasta superficie utilizando una red bongo $(250 \mu \mathrm{m}$ ojo de malla, $30 \mathrm{~cm}$ diámetro, flujómetro $\mathrm{GO}$ ). La duración de los arrastres para recolectar el zooplancton fue de 8 minutos a una velocidad promedio de $7 \mathrm{~km} / \mathrm{h}$. Tanto las muestras de fitoplancton como las de zooplancton fueron preservadas con formalina al $4 \%$.

Análisis de laboratorio: Se realizó la identificación taxonómica del fitoplancton hasta el nivel de género, mediante la revisión de $1 \mathrm{ml}$ de muestra homogenizada en una cámara Sedgwick-Rafter. Se siguieron las guías taxonómicas de Jiménez (1983) y Tomas (1993, 1997), y la validez actual de la nomenclatura se corroboró en la base de datos AlgaeBase (Guiry \& Guiry, 2013). Se estimó la abundancia de 30 grupos taxonómicos de zooplancton utilizando como referencia las guías de identificación de Boltovskoy (1999). Para establecer la abundancia de cada grupo taxonómico, se realizaron conteos de una fracción de la muestra recolectada con la red bongo y se estandarizó a ind $/ 100 \mathrm{~m}^{3}$ considerando el volumen de agua filtrado en cada estación. Finalmente, se cuantificó la producción secundaria a partir de la biomasa del zooplancton (peso seco) siguiendo el protocolo propuesto por Postel, Fock \& Hagen (2000). Para esto se filtró el exceso de agua de las muestras recolectadas en uno de los colectores de la red bongo, utilizando un sistema de vacío y filtros Whatman $\mathrm{GF} / \mathrm{F}$ ( $7 \mathrm{~cm}$ de diámetro, poro de $0.7 \mu \mathrm{m})$. Posteriormente, las muestras se secaron en un horno eléctrico $(24 \mathrm{~h}$ a $60^{\circ} \mathrm{C}$ ), y después se pesaron en una balanza analítica RADWAG $( \pm 0.0001 \mathrm{~g})$.

Análisis de datos: Se construyeron mapas de contorno y mapas de distribución para describir la variación espacial de la transparencia del agua, la producción secundaria medida en función de la biomasa seca y la abundancia de los grupos dominantes del zooplancton en 
la zona de estudio. Se estableció la frecuencia de registro para cada género de fitoplancton durante cada periodo y se realizó un análisis no paramétrico de Mann-Whitney para evaluar la variación de las variables biológicas (biomasa y grupos del zooplancton) e hidrográficas (temperatura, salinidad, oxígeno disuelto, profundidad de la termoclina y transparencia) entre los dos periodos de estudio, debido a que no se cumplieron con los supuestos de homogeneidad de varianzas y/o normalidad. Finalmente, se evaluó el grado de asociación entre la abundancia y biomasa del zooplancton con las variables físico-químicas utilizando un análisis de correlación de Spearman. Todos los análisis estadísticos se realizaron con el programa computacional STATISTICA 7.0 (StatSoft, 2004).

\section{RESULTADOS}

Hidrografía: En ambos periodos de estudio la capa superficial de la columna de agua $(0-10 \mathrm{~m})$ fue cálida $\left(>25^{\circ} \mathrm{C}\right)$ y presentó poca variación vertical $\left(\mathrm{VVT}_{\text {octubre }}=0.2^{\circ} \mathrm{C}\right.$, $\mathrm{VVT}_{\text {marzo }}=0.7^{\circ} \mathrm{C}$ ). Sin embargo a $30 \mathrm{~m}$ de profundidad la temperatura fue significativamente menor durante marzo, siendo la profundidad de la termoclina (isoterma de $20^{\circ} \mathrm{C}$ ) durante este periodo de $15 \pm 2 \mathrm{~m}$ mientras que en octubre fue de $36 \pm 2.7 \mathrm{~m}$ (Cuadro 1).

La salinidad entre 0-30 $\mathrm{m}$ de profundidad fue significativamente menor durante octubre que durante marzo en las tres profundidades evaluadas (Cuadro 1). Sin embargo, durante octubre el mayor rango de variación

CUADRO 1

Mediana, mínimo (Min) y máximo (Max) de la isoterma de $\operatorname{los} 20^{\circ} \mathrm{C}$, la temperatura, la salinidad, el oxígeno disuelto y la transparencia en isla Gorgona durante octubre de 2010 (época de lluvias) y marzo de 2011 (época seca).

TABLE 1

Median, minimum (Min) and maximum (Max) of the $20^{\circ} \mathrm{C}$ isotherm, temperature, salinity, and dissolved oxygen on Isla Gorgona during October 2010 (wet season) and March 2011 (dry season).

\begin{tabular}{|c|c|c|c|c|c|c|c|c|c|}
\hline & \multicolumn{4}{|c|}{ Octubre 2010} & \multicolumn{4}{|c|}{ Marzo 2011} & \multirow{2}{*}{$\mathrm{p}$ valor } \\
\hline & $\mathrm{n}$ & Min & Max & Mediana & $\mathrm{n}$ & Min & Max & Mediana & \\
\hline \multicolumn{10}{|c|}{ Isoterma $20^{\circ} \mathrm{C}(\mathrm{m})$} \\
\hline & 11 & 29.0 & 42.0 & 36.0 & 21 & 11.0 & 27.0 & 15.0 & $<0.001$ \\
\hline \multicolumn{10}{|c|}{ Temperatura $\left({ }^{\circ} \mathrm{C}\right)$} \\
\hline $2 \mathrm{~m}$ & 21 & 26.4 & 27.2 & 27.0 & 24 & 27.5 & 28.3 & 27.8 & $<0.001$ \\
\hline $10 \mathrm{~m}$ & 21 & 26.5 & 27.0 & 26.9 & 23 & 25.0 & 27.7 & 27.1 & $<0.001$ \\
\hline $30 \mathrm{~m}$ & 16 & 16.7 & 27.0 & 25.5 & 19 & 15.2 & 17.7 & 15.6 & $<0.001$ \\
\hline \multicolumn{10}{|c|}{ Salinidad (UPS) } \\
\hline $2 \mathrm{~m}$ & 21 & 27.5 & 28.6 & 28.0 & 24 & 27.2 & 30.9 & 29.6 & $<0.001$ \\
\hline $10 \mathrm{~m}$ & 21 & 27.7 & 28.8 & 28.2 & 23 & 26.2 & 32.5 & 31.4 & $<0.001$ \\
\hline $30 \mathrm{~m}$ & 16 & 28.8 & 34.8 & 30.2 & 19 & 34.4 & 34.9 & 34.8 & $<0.001$ \\
\hline \multicolumn{10}{|c|}{ Oxígeno Disuelto (ml/1) } \\
\hline $2 \mathrm{~m}$ & 19 & 4.2 & 5.2 & 4.8 & 24 & 4.2 & 4.9 & 4.3 & $<0.001$ \\
\hline $10 \mathrm{~m}$ & 17 & 4.3 & 5.1 & 4.7 & 24 & 3.2 & 4.5 & 4.3 & $<0.001$ \\
\hline $30 \mathrm{~m}$ & 12 & 3.8 & 4.8 & 4.3 & 20 & 1.7 & 2.7 & 2.1 & $<0.001$ \\
\hline \multicolumn{10}{|c|}{ Transparencia (m) } \\
\hline & 24 & 4.0 & 9.0 & 7.0 & 23 & 6.1 & 15.0 & 11.5 & $<0.001$ \\
\hline
\end{tabular}

p valor: Mann-Whitney al comparar variables entre las dos épocas; n: número de datos.

$\mathrm{p}$ value: Mann-Whitney values between seasons; $\mathrm{n}$ : number of data. 
de la salinidad se registró a $30 \mathrm{~m}$ de profundidad $\left(\mathrm{VVS}_{\text {octubre }}=6.0 \mathrm{UPS}\right)$, mientras que durante marzo la mayor variación de la salinidad se registró a solo $10 \mathrm{~m}$ de profundidad $\left(\mathrm{VVS}_{\mathrm{marzo}}=6.3 \mathrm{UPS}\right)$.

La concentración del oxígeno disuelto fue significativamente menor durante marzo en las tres profundidades evaluadas (Cuadro 1), con registros de $1.7 \mathrm{ml} / 1$ a $30 \mathrm{~m}$ en el sector occidental del PNN Gorgona. Por otro lado, la transparencia de la columna de agua fue significativamente menor en octubre (Cuadro 1), detectándose un núcleo de baja transparencia en el sector oriental de la isla, mientras que durante marzo la menor transparencia se registró en el sector sur del área de estudio probablemente como consecuencia de la baja profundidad en este sector (Fig. 2).

Fitoplancton: Se registraron un total de 61 géneros de fitoplancton para los dos periodos analizados, con un mayor número de géneros en marzo (56, 8 exclusivos) que en octubre (50, 4 exclusivos) (Cuadro 2). Las diatomeas fueron el grupo dominante en número de géneros tanto en octubre (78\%) como en marzo $(77 \%)$, siendo géneros representativos por su frecuencia de observación en las placas de identificación durante los dos periodos de muestreo, Bacteriastrum, Chaetoceros, Coscinodiscus,
Guinardia, Rhizosolenia y Skeletonema (Cuadro 2). Sin embargo, durante octubre también se destacaron por su alta frecuencia de registro (frecuencia=1.0) Asterionellopsis, Asteromphalus, Asteroplanus, Aulacoseira, Ditylum, Odontella y Thallasionema, mientras que durante marzo se destacaron Leptocylindrus y Hemiaulus (Cuadro 2).

Después de las diatomeas, los dinoflagelados fueron el segundo grupo en presentar el mayor número de géneros (18\% octubre, $21 \%$ marzo). Aunque ningún género de dinoflagelado tuvo una frecuencia de registro del $100 \%$ durante octubre o durante marzo, los géneros de mayor frecuencia de registro durante ambos periodos de estudio fueron Ceratium y Gonyaulax (Cuadro 2). Los silicoflagelados fueron un componente menor del fitoplancton para la isla en términos del número de géneros (Cuadro 2), siendo el género Dictyocha el que presentó una mayor frecuencia de registro, durante octubre 2010 (Cuadro 2).

Zooplancton: La biomasa del zooplancton (peso seco) varió entre 1.1 y $28.0 \mathrm{mg} / \mathrm{m}^{3}$ durante octubre (mediana: $7.5 \mathrm{mg} / \mathrm{m}^{3}$ ) y entre 1.9 y $168.7 \mathrm{mg} / \mathrm{m}^{3}$ durante marzo (mediana: $16.2 \mathrm{mg} / \mathrm{m}^{3}$ ), siendo los valores registrados durante marzo significativamente mayores (Mann-Whitney, $\mathrm{p}<0.001$ ). Espacialmente, la
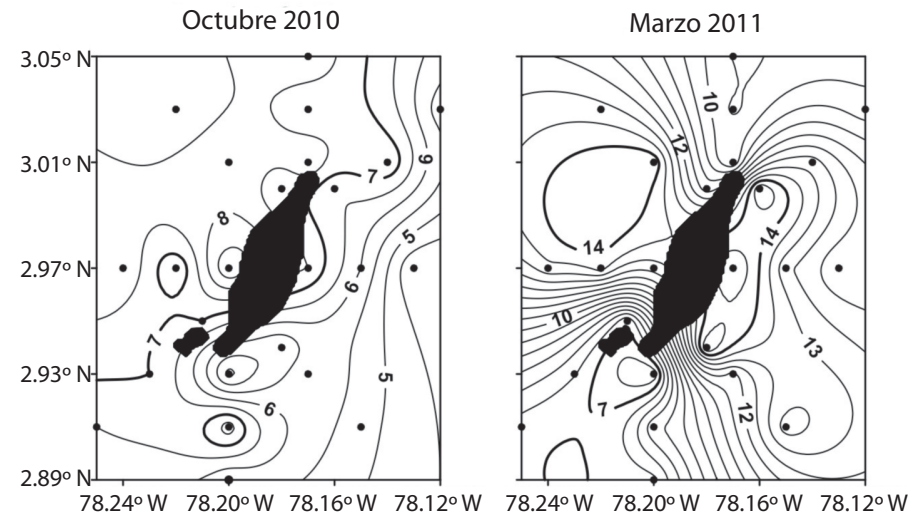

Fig. 2. Variación espacial y temporal de la transparencia $(\mathrm{m})$ en la columna de agua en isla Gorgona durante octubre de 2010 (época de lluvias) y marzo de 2011 (época seca). Isolíneas principales cada $1 \mathrm{~m}$, isolíneas secundarias cada $0.5 \mathrm{~m}$.

Fig. 2. Spatial distribution and temporal variation of water column transparency $(\mathrm{m})$ in Isla Gorgona during October 2010 (wet season) and March 2011 (dry season). Main isolines at $1 \mathrm{~m}$, secondary isolines at $0.5 \mathrm{~m}$. 
CUADRO 2

Composición y frecuencia de registro de los géneros del fitoplancton en isla Gorgona durante octubre $2010(\mathrm{n}=11)$ y marzo $2011(\mathrm{n}=24)$.

TABLE 2

Composition and frequency of phytoplankton genera in Isla Gorgona, during October $2010(\mathrm{n}=11)$ and March $2011(\mathrm{n}=24)$.

\begin{tabular}{|c|c|c|c|c|c|}
\hline & Oct. & Mar. & & Oct. & Mar. \\
\hline Diatomeas & & & Trachyneis & 0.0 & 0.04 \\
\hline Familia Asterolampraceae & & & Familia Paraliaceae & & \\
\hline Asteromphalus & 1.0 & 0.2 & Paralia & 0.3 & 0.0 \\
\hline Familia Aulacoseiraceae & & & Familia Protoperidiniaceae & & \\
\hline Aulacoseira & 1.0 & 0.9 & Protoperidinium & 0.5 & 0.5 \\
\hline Familia Bacillariaceae & & & Preperidinium & 0.0 & 0.04 \\
\hline Nitzschia & 0.9 & 0.2 & Familia Pyrophacaceae & & \\
\hline Pseudo-nitzschia & 0.9 & 0.3 & Pyrophacus & 0.7 & 0.3 \\
\hline Familia Biddulphiaceae & & & Familia Pleurosigmataceae & & \\
\hline Trigonium & 0.7 & 0.5 & Pleurosigma & 0.1 & 0.3 \\
\hline Familia Catenulaceae & & & Familia Protoperidiniaceae & & \\
\hline Amphora & 0.0 & 0.4 & Diplopelta & 0.1 & 0.0 \\
\hline Familia Chaetoceraceae & & & Familia Rhizosoleniaceae & & \\
\hline Bacteriastrum & 1.0 & 1.0 & Dactyliosolen & 0.0 & 0.1 \\
\hline Chaetoceros & 1.0 & 1.0 & Guinardia & 1.0 & 1.0 \\
\hline Familia Cocconeidaceae & & & Pseudosolenia & 0.8 & 0.7 \\
\hline Cocconeis & 0.0 & 0.04 & Proboscia & 1.0 & 1.0 \\
\hline Familia Corethraceae & & & Rhizosolenia & 1.0 & 1.0 \\
\hline Corethron & 0.8 & 1.0 & Familia Skeletonemaceae & & \\
\hline Familia Coscinodiscaceae & & & Detonula & 0.6 & 0.1 \\
\hline Coscinodiscus & 1.0 & 1.0 & Skeletonema & 1.0 & 1.0 \\
\hline Familia Diploneidaceae & & & Familia Stauroneidaceae & & \\
\hline Diploneis & 0.0 & 0.1 & Meuniera & 0.3 & 0.4 \\
\hline Familia Eunotiaceae & & & Familia Stephanodiscaceae & & \\
\hline Eunotia & 0.0 & 0.04 & Cyclotella & 0.7 & 0.5 \\
\hline Familia Fragilariaceae & & & Familia Stephanopyxidaceae & & \\
\hline Asterionellopsis & 1.0 & 0.2 & Stephanopyxis & 0.8 & 0.5 \\
\hline Asteroplanus & 1.0 & 0.0 & Familia Thalassionemataceae & & \\
\hline Opephora & 0.4 & 0.3 & Lioloma & 0.2 & 0.6 \\
\hline Familia Hemiaulaceae & & & Thalassionema & 1.0 & 0.5 \\
\hline Climacodium & 0.4 & 0.8 & Thalassiothrix & 0.9 & 0.9 \\
\hline Eucampia & 0.5 & 0.7 & Familia Thalassiosiraceae & & \\
\hline Hemiaulus & 0.9 & 1.0 & Planktoniella & 0.1 & 0.0 \\
\hline Familia Hemidiscaceae & & & Thalassiosira & 0.2 & 0.8 \\
\hline Actinocyclus & 0.2 & 0.3 & Familia Triceratiaceae & & \\
\hline Familia Lauderiaceae & & & Odontella & 1.0 & 0.9 \\
\hline Lauderia & 0.7 & 0.8 & Triceratium & 0.0 & 0.04 \\
\hline \multicolumn{6}{|l|}{ Familia Leptocylindraceae } \\
\hline Leptocylindrus & 0.8 & 1.0 & Dinoflagelados & & \\
\hline Familia Lithodesmiaceae & & & Familia Amphisoleniaceae & & \\
\hline Ditylum & 1.0 & 0.9 & Amphisolenia & 0.4 & 0.1 \\
\hline Lithodesmium & 0.3 & 0.1 & Familia Ceratiaceae & & \\
\hline
\end{tabular}


CUADRO 2 (Continuación) / TABLE 2 (Continued)

\begin{tabular}{|c|c|c|c|c|c|}
\hline & Oct. & Mar. & & Oct. & Mar. \\
\hline Familia Melosiraceae & & & Ceratium & 0.7 & 0.8 \\
\hline Melosira & 0.3 & 0.04 & Familia Ceratocoryaceae & & \\
\hline Familia Naviculaceae & & & Ceratocorys & 0.1 & 0.2 \\
\hline Navicula & 0.0 & 0.1 & Familia Goniodomataceae & & \\
\hline Familia Dinophysiaceae & & & Goniodoma & 0.1 & 0.04 \\
\hline Dinophysis & 0.1 & 0.1 & & & \\
\hline Ornithocercus & 0.0 & 0.1 & Silicoflagelados & & \\
\hline Familia Gonyaulacaceae & & & Familia Dictyochaceae & & \\
\hline Gonyaulax & 0.4 & 0.4 & Dictyocha & 0.8 & 0.04 \\
\hline Protoceratium & 0.0 & 0.04 & Mesocena & 0.1 & 0.0 \\
\hline \multicolumn{6}{|l|}{ Familia Podolampadaceae } \\
\hline Podolampas & 0.1 & 0.3 & & & \\
\hline
\end{tabular}

distribución de la biomasa del zooplancton fue homogénea durante octubre, donde en el 96\% de las estaciones los valores registrados fueron menores a $25 \mathrm{mg} / \mathrm{m}^{3}$. Durante marzo, estos mismos valores se registraron en el $63 \%$ de las estaciones, debido a la presencia de núcleos con valores mayores $\left(>50 \mathrm{mg} / \mathrm{m}^{3}\right)$ en dos estaciones del sector oriental y tres estaciones del sector occidental de la isla (Fig. 3). La abundancia del zooplancton presentó alta variación espacial durante los dos periodos evaluados, registrándose concentraciones mayores a $450 \mathrm{ind} / \mathrm{m}^{3}$ en el $13 \%$ de las estaciones durante octubre y en el $42 \%$ de las estaciones durante marzo (Fig. 3 ).

La abundancia total del zooplancton fue significativamente menor en octubre (mediana: 254ind $/ \mathrm{m}^{3}$ ) que en marzo (mediana: 432ind/ $\mathrm{m}^{3}$ ), debido a que 15 de los 30 grupos cuantificados presentaron abundancias significativamente mayores durante marzo (Cuadro 3). En contraste, sólo cuatro grupos (larvas de decápodos, larvas de cirripedios, isópodos, y larvas de estomatópodos) presentaron abundancias significativamente mayores durante octubre (Cuadro 3). Aunque la abundancia de los grupos del zooplancton varió entre los periodos de muestreo, en ambos periodos los copépodos presentaron la mayor contribución (68\% octubre, $69 \%$ marzo). Entre los otros grupos cuantificados se destacan por su contribución a la abundancia de la comunidad, las larvas de decápodos ( $8 \%)$, los quetognatos
(5\%), los ostrácodos $(4 \%)$ y las apendicularias (3\%) durante octubre, así como los quetognatos $(6 \%)$, las larvas de bivalvos (4\%), las apendicularias $(3 \%)$ y las larvas de decápodos $(3 \%)$ durante marzo (Cuadro 3).

La variación espacial de la abundancia de los 12 primeros grupos taxonómicos de zooplancton en los que se detectaron diferencias significativas entre los dos periodos de estudio se presenta en la Fig. 4 y Fig. 5. Durante octubre, algunos grupos como los copépodos, pterópodos y medusas presentaron núcleos con mayores abundancias hacia el sector occidental de la isla, mientras que los cirripedios presentaron núcleos de mayores abundancias hacia el sector oriental. Durante marzo, los quetognatos, pterópodos y anfípodos presentaron núcleos con mayores abundancias hacia el sector oriental, mientras que organismos meroplanctónicos, como los decápodos, presentaron núcleos con mayores abundancias hacia el sector occidental de la isla tanto en octubre como en marzo, específicamente en las estaciones cercanas a la zona de Montañitas (Fig. 4, Fig. 5).

Relación entre el zooplancton y la hidrografía: La abundancia total del zooplancton se correlacionó directamente con la transparencia e inversamente con la concentración de oxígeno disuelto a $10 \mathrm{~m}$, aunque estas correlaciones fueron débiles (Cuadro 4). De otro lado, la 

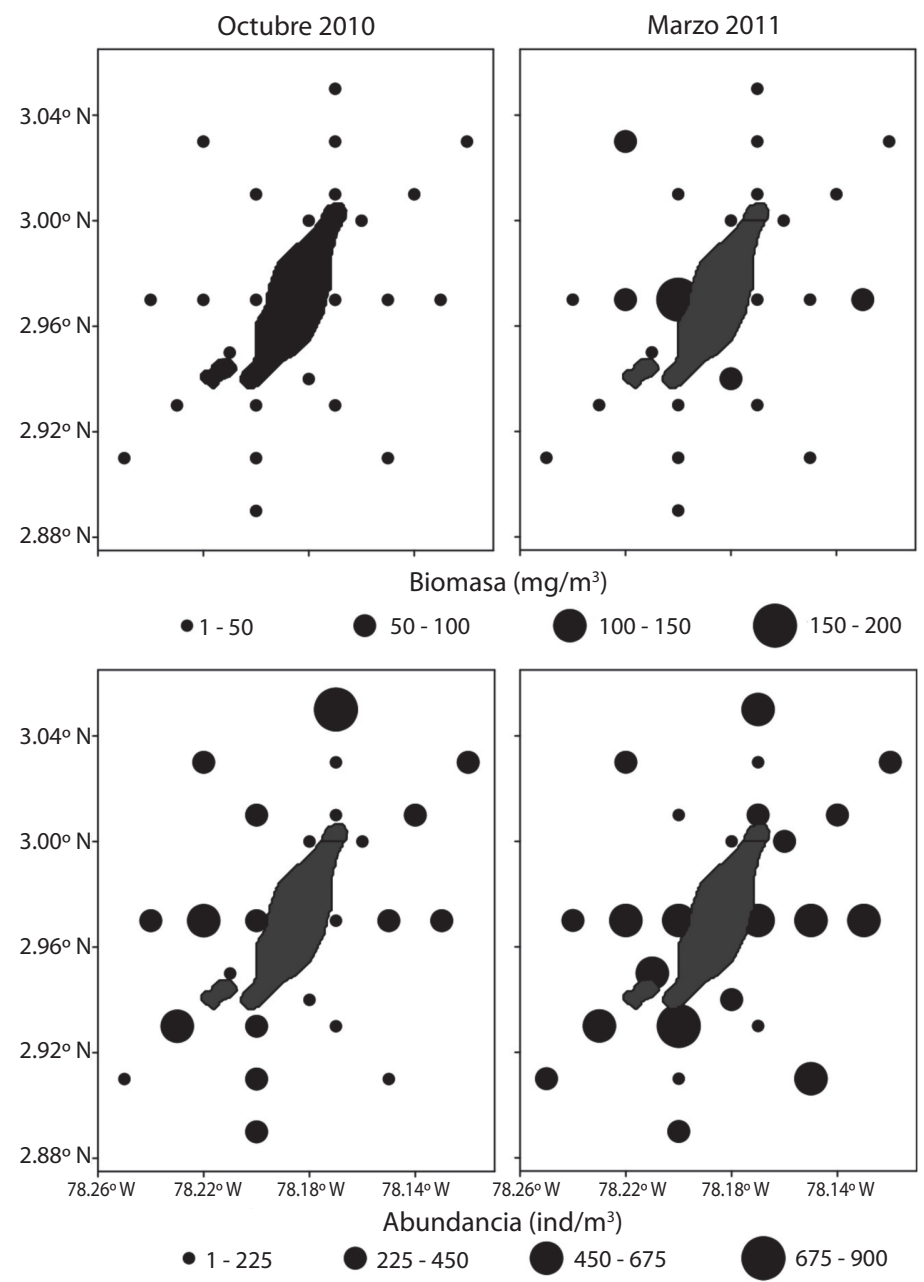

Fig. 3. Biomasa (peso seco $\mathrm{mg} / \mathrm{m}^{3}$ ) y abundancia del zooplancton (ind $/ \mathrm{m}^{3}$ ) en isla Gorgona durante octubre de 2010 (época de lluvias) y marzo de 2011 (época seca).

Fig. 3. Zooplankton biomass (dry weight $\mathrm{mg} / \mathrm{m}^{3}$ ) and abundance (ind $/ \mathrm{m}^{3}$ ) on Isla Gorgona during October 2010 (wet season) and March 2011 (dry season).

biomasa presentó correlaciones significativas directamente proporcionales con la temperatura superficial, la salinidad a $10 \mathrm{~m}$ y la transparencia, e inversamente proporcionales con la concentración de oxígeno en superficie $(1 \mathrm{~m})$ y a $10 \mathrm{~m}$ de profundidad (Cuadro 4). Solamente los Bryozoa, Foraminífera e Isópoda presentaron correlaciones fuertes $\left(r_{s}>0.75\right)$ y significativas con alguno de los parámetros oceanográficos evaluados (Cuadro 4). Otros grupos de alta importancia ecológica para el sistema pelágico, como copépodos, quetognatos, apendicularias, salpas, eufáusidos y anfípodos, así como grupos de interés para la conservación como las larvas decápodos, larvas gastrópodos y las larvas y huevos de peces, presentaron asociaciones entre débiles y moderadas con parámetros ambientales particulares (Cuadro 4).

\section{DISCUSIÓN}

Las variaciones estacionales en las condiciones hidrográficas de la columna de agua 
CUADRO 3

Abundancia relativa (\%) y mediana de los grandes grupos del zooplancton en isla Gorgona durante octubre $2010(n=24)$ y marzo $2011(n=24)$.

TABLE 3

Relative abundance (\%) and median of the major taxonomic groups of zooplankton on Isla Gorgona during October $2010(n=24)$ and March $2011(n=24)$.

\begin{tabular}{|c|c|c|c|c|c|c|}
\hline & \multicolumn{2}{|c|}{ Octubre 2010} & \multicolumn{2}{|c|}{ Marzo 2011} & \multirow[b]{2}{*}{$\mathrm{U}$} & \multirow[b]{2}{*}{$\mathrm{p}$} \\
\hline & $\%$ & Mediana & $\%$ & Mediana & & \\
\hline Copepoda & 68.11 & 17656 & 68.58 & 29395 & 183 & 0.03 \\
\hline Chaetognata & 5.35 & 1293 & 6.27 & 2209 & 165 & 0.01 \\
\hline Decapoda & 7.64 & 1805 & 2.92 & 543 & 119 & $<0.01$ \\
\hline Apendicularia & 3.12 & 615 & 3.29 & 1199 & 216 & 0.14 \\
\hline Bivalvia & 1.41 & 355 & 4.08 & 1324 & 60 & $<0.01$ \\
\hline Ostracoda & 3.70 & 635 & 1.77 & 208 & 213 & 0.12 \\
\hline Cladocera & 2.43 & 173 & 1.61 & 503 & 213 & 0.12 \\
\hline Gastropoda & 2.17 & 503 & 1.58 & 464 & 280 & 0.87 \\
\hline Pteropoda & 1.18 & 291 & 1.60 & 471 & 164 & 0.01 \\
\hline Siphonophora & 0.49 & 132 & 1.46 & 463 & 20 & $<0.01$ \\
\hline Amphipoda & 0.48 & 102 & 1.02 & 367 & 102 & $<0.01$ \\
\hline Medusa & 0.42 & 101 & 0.92 & 416 & 101 & $<0.01$ \\
\hline Polychaeta & 0.27 & 61 & 0.98 & 356 & 56 & $<0.01$ \\
\hline Bryozoa & 0.02 & $<0.1$ & 1.13 & 259 & 4 & $<0.01$ \\
\hline Doliola & 0.34 & 58 & 0.77 & 272 & 75 & $<0.01$ \\
\hline Cirripedia & 0.80 & 163 & 0.14 & 23 & 101 & $<0.01$ \\
\hline Larva pluteus & 0.12 & 19 & 0.49 & 108 & 69 & $<0.01$ \\
\hline Isopoda & 0.70 & 177 & 0.01 & $<0.1$ & 29 & $<0.01$ \\
\hline Huevo Pez & 0.38 & 35 & 0.24 & 14 & 221 & 0.17 \\
\hline Foraminifera & 0.00 & $<0.1$ & 0.37 & 134 & 13 & $<0.01$ \\
\hline Stomatopoda & 0.42 & 62 & 0.05 & $<0.1$ & 89 & $<0.01$ \\
\hline Heteropoda & 0.16 & 31 & 0.19 & 42 & 232 & 0.24 \\
\hline Euphausiacea & 0.22 & $<0.1$ & 0.12 & 35 & 158 & 0.01 \\
\hline Larva Pez & 0.02 & $<0.1$ & 0.22 & 78 & 23 & $<0.01$ \\
\hline Ctenophora & 0.04 & 3 & 0.05 & 19 & 209 & 0.10 \\
\hline Salpida & 0.01 & $<0.1$ & 0.06 & $<0.1$ & 200 & 0.07 \\
\hline Larva Brachiopoda & 0.00 & $<0.1$ & 0.05 & 17 & 140 & $<0.01$ \\
\hline Mysidacea & 0.01 & $<0.1$ & 0.02 & $<0.1$ & 288 & 0.99 \\
\hline Cephalopoda & 0.00 & 0 & 0.01 & $<0.1$ & 264 & 0.62 \\
\hline \multirow[t]{2}{*}{ Larva Brachiolaria } & 0.00 & 0 & 0.01 & $<0.1$ & 252 & 0.46 \\
\hline & $100 \%$ & & $100 \%$ & & & \\
\hline
\end{tabular}

p valor: Mann-Whitney al comparar variables entre las dos épocas; $n$ : número de datos.

$\mathrm{p}$ value: Mann-Whitney values between seasons; $n$ : number of data.

suelen reflejarse en cambios en la composición y/o abundancia de los organismos planctónicos, ya que estos por su historia de vida responden rápidamente ante los pulsos presentados en las condiciones ambientales (Ayala et al., 1996; Bednarski \& Morales-Ramírez, 2004;
Fernández-Álamo \& Färber-Lorda, 2006; Pennington et al., 2006). En isla Gorgona, la variación estacional registrada para la hidrografía con el ingreso de aguas frías, salinas y con bajas concentraciones de oxígeno disuelto durante la época seca (marzo de 2011), y con 

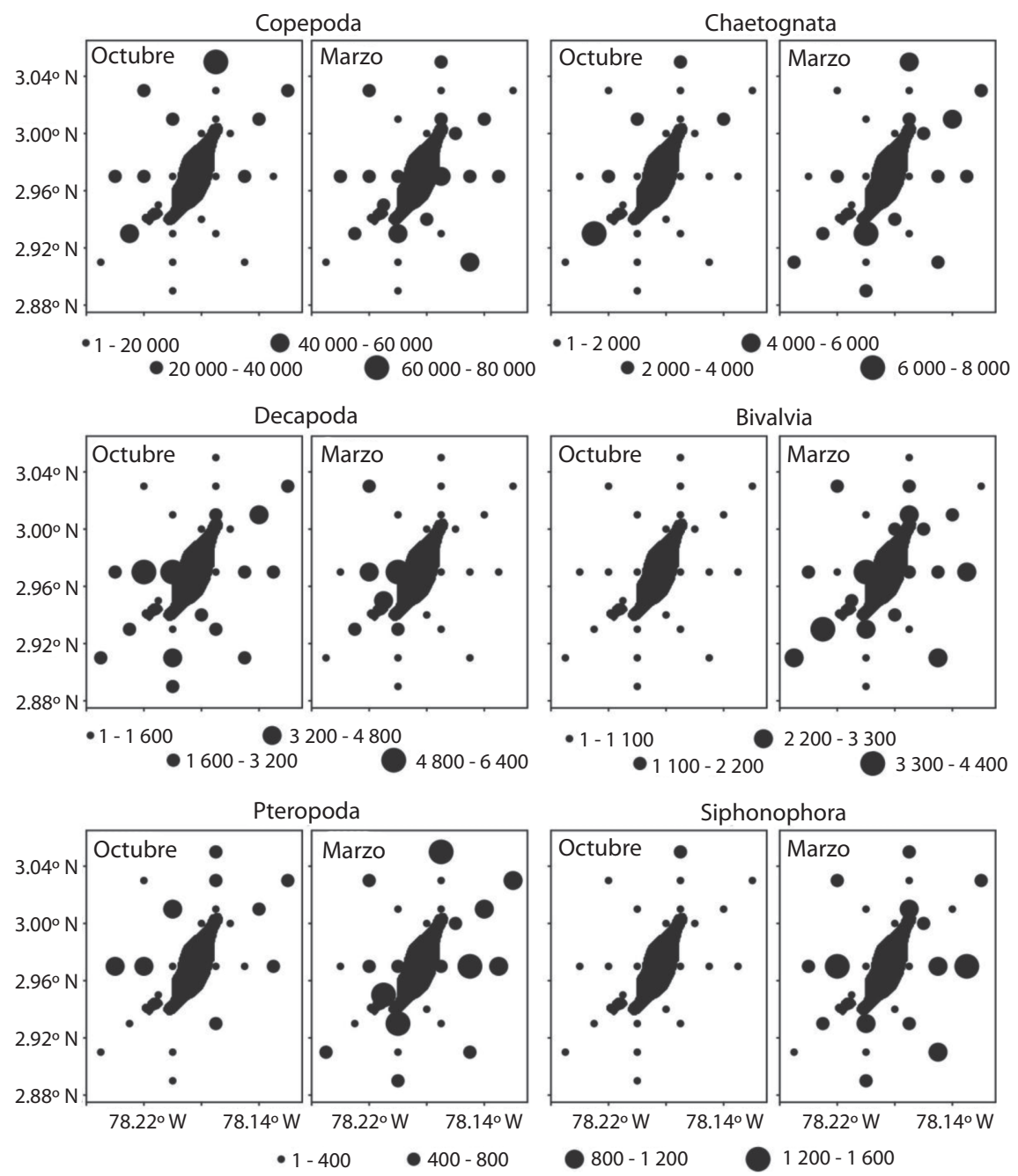

Fig. 4. Distribución de la abundancia de seis grupos del zooplancton (ind $/ 100 \mathrm{~m}^{3}$ ) en isla Gorgona durante octubre de 2010 (época de lluvias) y marzo de 2011 (época seca). Nota: la escala de abundancia varía entre los grupos.

Fig. 4. Distribution of the abundance of six zooplankton groups (ind $/ 100 \mathrm{~m}^{3}$ ) on Isla Gorgona during October 2010 (wet season) and March 2011 (dry season). Note: the scale of abundance varies between groups.

aguas cálidas y de bajas salinidades durante la época de lluvias (octubre de 2010), son consecuentes con lo reportado previamente para por Díaz et al. (2001), Giraldo (2008) y Giraldo et al. (2008) para esta localidad, y se reflejaron en cambios en la abundancia y biomasa del zooplancton.

Aunque los organismos planctónicos constituyen los principales productores primarios y secundarios de la columna de agua, y por ende los cambios en su composición, abundancia, y biomasa modulan la transferencia de energía hacia los niveles tróficos superiores, pocos estudios han evaluado la variación estacional del plancton en isla Gorgona y en general en el Pacífico colombiano. De acuerdo con lo reportado por Soto et al. (2001) para isla Gorgona y por D’Croz, Del Rosario \& Gómez (1991) para la Bahía de Panamá, región con estacionalidad similar a la de Gorgona, las mayores 

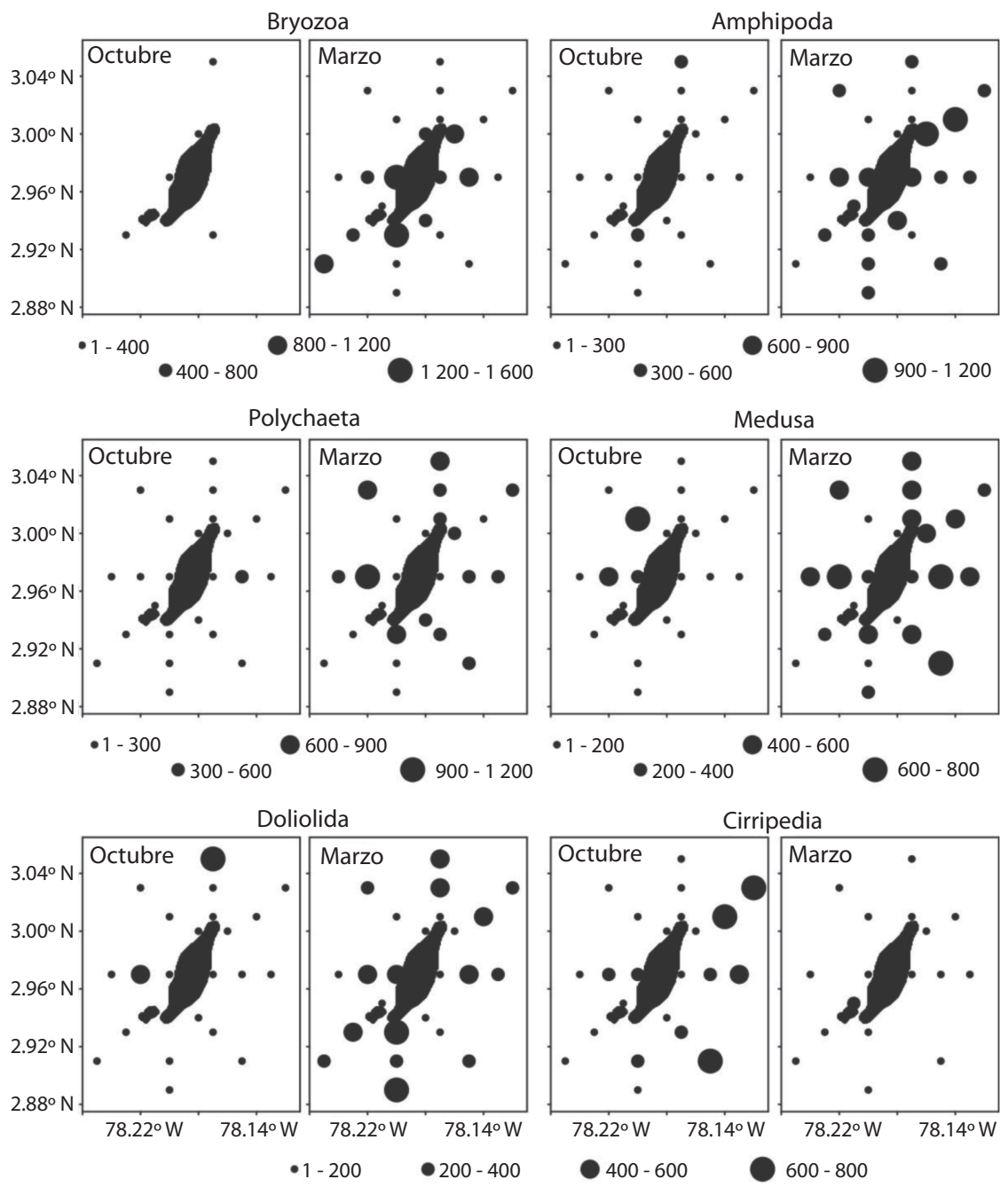

Fig. 5. Distribución de la abundancia de seis grupos del zooplancton (ind $/ 100 \mathrm{~m}^{3}$ ) en isla Gorgona durante octubre de 2010 (época de lluvias) y marzo de 2011 (época seca). Nota: la escala de abundancia varía entre los grupos.

Fig. 5. Distribution of the abundance of six zooplankton groups (ind $/ 100 \mathrm{~m}^{3}$ ) on Isla Gorgona during October 2010 (wet season) and March 2011 (dry season). Note: the scale of abundance varies between groups.

abundancias de fitoplancton se registran durante marzo. Por lo tanto, es de esperar que durante esta época el fitoplancton presente en el área marina protegida del PNN Gorgona responda al proceso estacional de surgencia que se presenta en una escala regional en la cuenca del Océano Pacífico colombiano (Rodríguez-Rubio et al., 2003; Devis-Morales et al., 2008).

De acuerdo con D'Croz \& O’Dea (2007), el incrementó de la abundancia del fitoplancton en la Bahía de Panamá durante la época seca (enero-abril) es el resultado de una mayor disponibilidad de nutrientes (nitrato y fosfato) como consecuencia del ingreso de aguas subsuperficiales por surgencia. En contraste, durante la época de lluvias (mayo-diciembre) la abundancia del fitoplancton disminuye por la ausencia de surgencia y el incremento en los niveles de precipitación, lo que reduce la concentración de los nutrientes en superficie. 
CUADRO 4

Coeficientes de correlación de Spearman $\left(\mathrm{r}_{\mathrm{s}}\right)$ entre el componente zooplanctónico y las variables oceanográficas superficiales del ambiente pelágico del PNN Gorgona durante octubre 2010 y marzo 2011.

TABLE 4

Spearman rank correlation $\left(\mathrm{r}_{\mathrm{s}}\right)$ between the zooplankton and surface oceanographic parameters of pelagic environment at PNN Gorgona during October 2010 and March 2011.

\begin{tabular}{|c|c|c|c|c|c|c|c|}
\hline & $\mathrm{T} 1 \mathrm{~m}$ & $\mathrm{~T} 10 \mathrm{~m}$ & $\mathrm{~S} 1 \mathrm{~m}$ & $\mathrm{~S} 10 \mathrm{~m}$ & OD1m & OD10m & Transp \\
\hline Asteroidea & 0.32 & -0.06 & -0.09 & 0.25 & -0.16 & -0.24 & 0.27 \\
\hline Amphipoda & 0.51 & 0.25 & 0.09 & 0.41 & -0.38 & -0.56 & 0.54 \\
\hline Apendicularia & 0.15 & 0.58 & -0.34 & -0.24 & 0.11 & 0.17 & -0.18 \\
\hline Bivalvia & 0.50 & 0.37 & 0.27 & 0.47 & -0.33 & -0.60 & 0.49 \\
\hline Brachiopoda & 0.47 & 0.18 & -0.03 & 0.25 & -0.36 & -0.30 & 0.23 \\
\hline Bryozoa & 0.73 & 0.47 & 0.33 & 0.58 & -0.47 & -0.75 & 0.59 \\
\hline Cephalopoda & -0.03 & 0.36 & 0.26 & 0.06 & -0.09 & -0.16 & -0.02 \\
\hline Chaetognata & 0.43 & 0.23 & -0.21 & 0.18 & -0.16 & -0.26 & 0.25 \\
\hline Cladocera & 0.09 & 0.17 & -0.10 & -0.19 & 0.13 & 0.12 & -0.08 \\
\hline Cirripedia & -0.55 & -0.08 & -0.37 & -0.55 & 0.33 & 0.44 & -0.69 \\
\hline Medusas & 0.48 & 0.22 & 0.13 & 0.45 & -0.35 & -0.44 & 0.57 \\
\hline Siphonophora & 0.72 & 0.32 & 0.19 & 0.56 & -0.52 & -0.70 & 0.58 \\
\hline Copepoda & 0.19 & 0.14 & 0.18 & 0.38 & -0.22 & -0.35 & 0.36 \\
\hline Ctenophora & 0.18 & -0.08 & 0.23 & 0.32 & -0.08 & -0.19 & 0.43 \\
\hline Decapoda & -0.30 & 0.17 & -0.39 & -0.53 & 0.46 & 0.45 & -0.49 \\
\hline Doliolida & 0.55 & 0.42 & 0.05 & 0.28 & -0.24 & -0.40 & 0.35 \\
\hline Euphausiacea & 0.35 & -0.06 & 0.13 & 0.41 & -0.51 & -0.61 & 0.45 \\
\hline Foraminifera & 0.76 & 0.48 & 0.17 & 0.51 & -0.53 & -0.72 & 0.53 \\
\hline Gastropoda & -0.01 & 0.12 & 0.09 & -0.04 & 0.13 & 0.03 & 0.06 \\
\hline Heteropoda & 0.14 & 0.04 & 0.22 & 0.31 & 0.06 & -0.14 & 0.23 \\
\hline Huevo Pez & -0.25 & 0.08 & -0.16 & -0.24 & 0.42 & 0.32 & -0.32 \\
\hline Isopoda & -0.82 & -0.62 & -0.19 & -0.44 & 0.59 & 0.77 & -0.49 \\
\hline Larva Pez & 0.71 & 0.44 & 0.25 & 0.57 & -0.41 & -0.67 & 0.60 \\
\hline Mysidacea & 0.04 & 0.03 & -0.21 & -0.25 & 0.09 & 0.12 & -0.27 \\
\hline Larva pluteus & 0.50 & 0.34 & 0.37 & 0.42 & -0.36 & -0.53 & 0.45 \\
\hline Ostracoda & -0.27 & -0.17 & 0.09 & 0.05 & 0.09 & 0.03 & -0.13 \\
\hline Polychaeta & 0.70 & 0.28 & 0.17 & 0.57 & -0.50 & -0.69 & 0.65 \\
\hline Pteropoda & 0.30 & 0.25 & -0.11 & 0.15 & -0.23 & -0.24 & 0.11 \\
\hline Stomatopoda & -0.45 & -0.18 & -0.34 & -0.49 & 0.33 & 0.46 & -0.60 \\
\hline Salpida & 0.16 & 0.10 & 0.26 & 0.20 & -0.17 & -0.23 & 0.06 \\
\hline Abundancia Total & 0.22 & 0.22 & 0.13 & 0.30 & -0.18 & -0.32 & 0.32 \\
\hline Biomasa Zoo & 0.55 & 0.25 & 0.18 & 0.48 & -0.50 & -0.67 & 0.48 \\
\hline
\end{tabular}

Coeficientes $\left(\mathrm{r}_{\mathrm{s}}\right)$ en negrita indican correlación significativa $(\mathrm{p}<0.05)$. Coefficients in bold (rs) indicate significant correlation $(\mathrm{p}<0.05)$.

$\mathrm{r}_{\mathrm{s}}<0.25$ : no hay correlación, $0.25<\mathrm{r}_{\mathrm{s}}<0.50$ : correlación débil, $0.50<\mathrm{r}_{\mathrm{s}}<0.75$ : correlación moderada, $\mathrm{r}_{\mathrm{s}}>0.75$ correlación fuerte.

Aunque en este trabajo no se estimó la concentración de nutrientes o la abundancia de fitoplancton en isla Gorgona, de acuerdo con Giraldo et al. (2008) y Giraldo et al. (2011), la surgencia estacional del Panama Bight y la variación del patrón local de precipitación son los dos principales forzantes ambientales que estarían modulando la productividad planctónica en esta localidad, por lo tanto, es de esperar que el patrón general de abundancia 
del fitoplancton en isla Gorgona sea similar al reportado para la Bahía de Panamá.

Géneros de fitoplancton como Chaetoceros, Bacteriastrum, Coscinodiscus, Skeletone$m a$, y Hemiaulus son comunes en las aguas costeras del Pacífico colombiano (Ramírez, Giraldo \& Tovar, 2006; Arteaga-Sogamoso, Rodríguez-Rubio \& Galeano, 2008; Giraldo \& Ramírez, 2010). Estos géneros han sido reportados previamente para isla Gorgona (Soto et al., 2001), y en otras localidades del POT como la Bahía de Panamá (Smayda, 1966; D’Croz et al., 1991) y el Golfo de Nicoya (BrugnoliOlivera \& Morales-Ramírez, 2001), en donde Chaetoceros y Skeletonema han sido identificados como los géneros dominantes.

$\mathrm{Al}$ igual que el fitoplancton, la abundancia y biomasa del zooplancton tiende a relacionarse significativamente con los cambios en las condiciones hidrográficas (e.g. disminución en la temperatura) o biológicas (aumento en la concentración de alimento) de un área en particular (Lo-Yat et al., 2011; Mackas et al., 2012). Durante el presente estudio, la abundancia del zooplancton se correlacionó positivamente solo con la salinidad superficial. Durante la época de lluvias en isla Gorgona, la salinidad de la parte superficial de la columna de agua disminuye (<28UPS), y en estas condiciones ambientales los organismos holoplanctónicos tienden a reducir su capacidad reproductiva, lo que se traduce en un descenso de su abundancia. En contraste, durante la época seca, el ingreso de agua subsuperfical (más fría, más salina, mayores concentraciones de nutrientes) al ambiente pelágico del PNN Gorgona (Giraldo \& Gutiérrez, 2007; Devis-Morales et al., 2008), promovería la productividad secundaria, incrementando la biomasa zooplanctónica disponible en esta localidad.

Soto et al. (2001) reportaron que el zooplancton en el ambiente pelágico del PNN Gorgona estuvo dominado en número por Chaetognata. Sin embargo, durante octubre 2010 y marzo de 2011 la comunidad zooplanctónica del PNN Gorgona estuvo dominada en número por Copepoda. Este resultado es consistente con lo registrado por Giraldo et al. (2011) para isla Gorgona, así como por otros estudios realizados en el Pacífico colombiano (Giraldo \& Gutiérrez, 2007; Murcia \& Giraldo, 2007) y en otras regiones del POT (e.g. Guzmán \& Obando, 1988; Bednarski \& Morales-Ramírez, 2004; Fernández-Álamo $\&$ Färber-Lorda, 2006). Desde un punto de vista trófico, es de esperar que un grupo de herbívoros-omnívoros como los copépodos sea dominante en número, ya que su variación en abundancia contribuye a estructurar la comunidad del fitoplancton mediante pastoreo, y del zooplancton mediante la transferencia de energía hacia niveles tróficos superiores.

La alta abundancia relativa de organismos del meroplancton, como larvas de decápodos y bivalvos, registradas durante esta investigación, posiblemente esté asociada con la gran variedad de especies bentónicas presentes en los ambientes intermareales y submareales de isla Gorgona, ya que su presencia en el plancton dependerá principalmente de la actividad reproductiva de los adultos (Highfield et al., 2010; Dias de Almeida-Fernandes, Quintanilla, Monteiro-Ribas, González-Rodríguez \& Countinho, 2012). En este sentido, ambientes rocosos intermareales y submareales presentes en la zona sur, norte y occidental de la isla, así como los arrecifes coralinos presentes en el sector oriental, representan hábitats típicos para organismos del bentos como decápodos, moluscos y poliquetos, entre otros, de ahí sus altas abundancias relativas en el ambiente pelágico.

Aunque isla Gorgona es una isla costera y se encuentra localizada en una zona tropical, las variaciones estacionales reportadas en la presente investigación, tanto para la hidrografía como para las comunidades planctónicas, reafirman que los procesos regionales y locales tienen una fuerte influencia sobre las condiciones ambientales y por ende, sobre las comunidades que allí habitan. Por esta razón, es de gran importancia que zonas protegidas como el PNN Gorgona cuenten con información básica de las variaciones temporales no solo de los parámetros ambientales como la temperatura, salinidad y oxígeno disuelto, sino también de la concentración del fitoplancton 
y zooplancton, ya que estos parámetros en su conjunto constituyen un sistema de alerta temprana ante procesos interanuales como el fenómeno ENOS, y de mayor escala como el cambio climático, que de acuerdo a su intensidad y duración pueden llegar a tener un efecto negativo en otros ecosistemas que son objetos de conservación de las áreas marinas protegidas, como el PNN Gorgona

\section{AGRADECIMIENTOS}

A Natalia Uribe, Andrés Cuellar, Katherine Mejía y Luis Felipe Piñeros por su colaboración durante la toma de muestras. A los funcionarios del PNN Gorgona por su apoyo logístico durante los muestreos. A la estación científica Henry von Prahl del PNN Gorgona por facilitar el espacio requerido para el procesamiento inicial de las muestras. La UAESPNN permitió el desarrollo de este proyecto en el PNN Gorgona bajo el permiso de investigación PIBD-DTSO-011-10. Dos evaluadores anónimos contribuyeron con sus sugerencias a mejorar el presente documento. Este trabajo fue financiado por Conservación Internacional Colombia, Fondo para la Acción Ambiental y la Niñez, Universidad del Valle y Fundación Squalus, y es un producto del proyecto "Evaluación del estado actual de los objetos de conservación faunísticos en Isla Gorgona: una aproximación holística a la valoración ecológica de PNN Gorgona".

\section{RESUMEN}

Se estableció la variación en la abundancia y biomasa del fitoplancton y zooplancton en el ambiente pelágico de isla Gorgona durante octubre 2010 (época lluviosa) y marzo 2011 (época seca), y se evaluó su grado de correlación con las condiciones hidrográficas de temperatura, salinidad, oxígeno disuelto y transparencia. Las aguas superficiales en los dos periodos fueron cálidas $\left(>26^{\circ} \mathrm{C}\right)$ y de bajas salinidades $(<31)$. A $30 \mathrm{~m}$ las aguas en octubre fueron homogéneas, mientras que en marzo fueron frías $\left(<18^{\circ} \mathrm{C}\right)$, salinas $(>34)$ y con bajas concentraciones de oxígeno $(3.0 \mathrm{ml} / 1)$. Se registraron 61 géneros de fitoplancton y 30 grupos de zooplancton, con mayores valores para la riqueza de géneros y abundancia del fitoplancton, y para la abundancia y biomasa del zooplancton en marzo que en octubre. Bacteriastrum, Chaetoceros, Coscinodiscus, Guinardia, Rhizosolenia y Skeletonema fueron los generous de fitoplancton más frecuentes durante ambos periodos de muestreo. Para el zooplancton los copépodos fueron el grupo dominante, seguido por los quetognatos y las apendicularias. Adicionalmente, la biomasa del zooplancton presentó una asociación positiva significativa con la temperatura superficial, la salinidad a $10 \mathrm{~m}$ y la transparencia, y negativa con la concentración de oxígeno disuelto en superficie y a $10 \mathrm{~m}$.

Palabras clave: fitoplancton, zooplancton, temperatura, salinidad, hidrografía, Pacífico Oriental Tropical, Colombia, Isla Gorgona.

\section{REFERENCIAS}

Amador, J. A., Alfaron, E. J. Lizano O. G. \& Magaña V. O. (2006). Atmospheric forcing of the eastern tropical Pacific: A review. Progress in Oceanography, 69: 101-142.

Arteaga-Sogamoso, E., Rodríguez-Rubio, E. \& Galeano, A. M. (2008). Distribución, abundancia y composición del fitoplancton y condiciones ambientales en la cuenca Pacífica colombiana, durante enero-febrero de 2007. Boletín Cientifico CCCP, 15: 105-122.

Ayala-Duval, E., Maldonado-Monroy, M. C., BecerrilMartínez, J. A., Ayala-Fernández, X. A., BarriosOrozco, V., García-Tamayo, D. T. \& Juárez-Ortiz, C. (1996). Spatial and temporal distribution of zooplankton biomass in the Guf of Tehuantepec, Mexico. Pacific Science, 50: 415-426.

Bednarski, M. \& Morales-Ramírez, A. (2004). Composition, abundance and distribution of macrozooplankton in Culebra Bay, Gulf of Papagayo, Pacific coast of Costa Rica and its value as bioindicator of pollution. Revista de Biología Tropical, 52: 105-119.

Blanco, J. F. (2009). The hydroclimatology of Gorgona Island: seasonal and ENSO-related patterns. Actualidades Biológicas, 31: 111-121.

Boltovskoy, D. (1999). (Ed). South Atlantic zooplankton. Backhuys Publishers, Holanda.

Brown, C. J., Fulton, E. A., Hobday, A. J., Matear, R. J., Possingham, H. P., Bulman, C., Christensen, V., Forrest, R. E., Gehrke, P. C., Gribble, N. A., Griffiths, S. P., Lozano-Montes, H., Martin, J. M., Metcalf, S., Okey, T. A., Watson, R. \& Richardson, A. J. (2010). Effects of climate-driven primary production change on marine food webs: implications for fisheries and conservation. Global Change Biology, 16:1194-1212.

Brugnoli-Olivera, E. \& Morales-Ramírez, A. (2001). La comunidad fitoplánctica de Punta Morales, Golfo de Nicoya, Costa Rica. Revista de Biología Tropical, 49: 11-17. 
D’Croz, L., Del Rosario, J. B. \& Gómez, J. A. (1991). Upwelling and phytoplankton in the Bay of Panama. Revista de Biología Tropical, 39: 233-241.

D’Croz, L. \& O’Dea, A. (2007). Variability in upwelling along the Pacific shelf of Panama and implications for the distribution of nutrients and chlorophyll. Estuarine, Coastal and Shelf Science, 73: 1-16.

Devis-Morales, A., Schneider, W., Montoya-Sánchez, R. A. \& Rodríguez-Rubio, E. (2008). Monsoon-like winds reverse oceanic circulation in the Panama Bight. Geophysical Research Letters, 35: L20607. doi: 10.1029/2008GL035172

Dias de Almeida-Fernandes, L., Quintanilla, J., MonteiroRibas, W., González-Rodríguez, E. \& Countinho, R. (2012). Journal of Plankton Research, 34 (3): 236-244.

Díaz, J. M., Pinzón J. H., Perdomo, A. M., Barrios, L. M. \& López-Victoria, M. (2001). Generalidades. In L. M. Barrios \& M. L. Victoria (Eds.), Gorgona marina: Contribución al conocimiento de una isla única (pp. 17-26). INVEMAR, Serie de Publicaciones especiales $N^{\circ}$ 7. Santa Marta, Colombia.

Escarria, E., Beltrán-León, B. S., Giraldo, A. \& Zapata, F. (2007). Ichthyoplankton in the Nacional Natural Park Isla Gorgona (Pacific Ocean of Colombia) during September 2005. Investigaciones Marinas, Valparaiso, $35: 127-133$.

Eslava, J. A. (1993). Climatología. In P. Leyva (Ed.), Colombia Pacifico (pp. 136-147). Tomo I. Fondo para la protección del medio ambiente, FEN, Santafé de Bogotá, Colombia.

Fernández-Álamo, M. A. \& Färber-Lorda, J. (2006). Zooplankton and the oceanography of the Eastern Tropical Pacific: A review. Progress in Oceanography, 69: 318-359.

Fiedler, P. C. \& Talley, L .D. (2006). Hydrography of the eastern tropical Pacific: A review. Progress in Oceanography, 69: 143-180.

Forsbergh, E. D. (1969). On the climatology, oceanography and fisheries of the Panama Bight. Inter-American Tropical Tuna Commission, 14: 49-385.

Giraldo, A. \& E. Gutiérrez. (2007). Composición taxonómica del zooplancton superficial en el Pacífico colombiano (Septiembre 2003). Investigaciones Marinas, Valparaíso, 35: 117-122.

Giraldo, A. (2008). Variabilidad espacial de temperatura, salinidad y transparencia en el ambiente pelágico del PNN Gorgona durante septiembre 2007 y marzo 2008. Boletín Científico. Centro de Investigaciones Oceanográficas e Hidrográficas del Caribe, 26: 157-163.

Giraldo, A., Rodríguez-Rubio, E. \& Zapata, F. (2008). Condiciones oceanográficas en Isla Gorgona, Pacífico
Oriental Tropical de Colombia. Latin American Journal of Aquatic Research, 36: 121-128.

Giraldo, A. \& Ramírez, D. G. (2010). Fitoplancton costero en Cabo Marzo y Punta Cruces, margen nororiental del Océano Pacífico colombiano. Boletín Cientifico. Centro de Investigaciones Oceanográficas e Hidrográficas del Caribe, 28: 172-202.

Giraldo, A., Valencia, B. \& Ramírez, D. G. (2011). Productividad planctónica y condiciones oceanográficas locales en Isla Gorgona durante julio 2006. Bol. Invest. Mar. Cost. 40: 185-201.

Giraldo, A. \& Valencia, B. (2012). Isla Gorgona: paraíso de biodiversidad y ciencia. Comité Editiorial Universidad del Valle. Cali, Colombia.

Guiry, M. D. \& Guiry, G. M. (2013). AlgaeBase. Worldwide electronic publication, National University of Ireland, Galway. Retrieved from: www.algaebase.org

Guzmán, H. M. \& Obando, V. L. (1988). Diversidad y abundancia diaria y estacional del zooplancton marino de la Isla del Caño, Costa Rica. Revista de Biología Tropical, 36: 139-150.

Highfield, J. M., Eloire, D., Conway, D. V., Lindeque, P. K., Attrill, M. J. \& Somerfield, P. J. (2010). Journal of Plankton Research, 32(5): 681-691.

Jiménez, R. (1983). Diatomeas y silicoflagelados del fitoplancton del golfo de Guayaquil. Acta Oceanográfica del Pacífico, 2: 193-281.

Lalli, C. M. \& Parsons, T. R. (1997). Biological oceanography: an introduction. Second edition. The Open University, Butterworth-Heinemann. Oxford, U.K.

Lo-Yat, A., Simpson, S. D., Meekan, M., Lecchini, D., Martínez, E. \& Galzin, R. (2011). Extreme climatic events reduce ocean productivity and larval supply in a tropical reef ecosystem. Global Change Biology, 17: 1695-1702.

Mackas, L., Greve, W., Edwards, M., Chiba, S., Tadokoro, K., Eloire, D., Mazzocchi, M. G., Batten, S., Richardson, A. J., Johnson, C., Head, E., Conversi, A. \& Peluso, T. (2012). Changing zooplankton seasonality in a changing ocean: Comparing time series of zooplankton phenology. Progress in Oceanography, 97:31-62.

Mann, K. H. \& Lazier, J. R. N. (2006). Dynamics of marine ecosystems: Biological-Physical Interactions in the Oceans. Blackwell Publishing. Oxford, U.K.

Miller, C. B. (2004). Biological oceanography. Blackwell Publishing. Oxford, U.K.

Murcia, M. \& Giraldo, A. (2007). Condiciones oceanográficas y composición del mesozooplancton en la zona oceánica del Pacífico colombiano, durante septiembre-octubre 2004. Boletín Científico CCCP, 14: 83-89. 
Pennington, J. T., Mahoney, K. L., Kuwahara, V. S., Kolber, D. D., Calienes. R. \& Chavez, F. P. (2006). Primary production in the Eastern Tropical Pacific: A review. Progress in Oceanography, 69: 285-317.

Postel, L., Fock, H. \& Hagen, W. (2000). Biomass and abundance. In R. Harris, P. Wiebe, J. Lenz, H.R. Skjoldal. \&. M. Huntley (Eds.). ICES zooplankton methodology manual (pp. 83-192). Academic Press. London, England.

Poveda, G. \& Mesa. O. (2000). On the existence of Lloró (the rainiest locality on earth): enhanced ocean-landatmosphere interaction by a low-level jet. Geophysical Research Letters, 27: 1675-1678.

Ramírez, D. G., Giraldo, A. \& Tovar, J. (2006). Producción primaria, biomasa y composición taxonómica del fitoplancton costero y oceánico en el Pacífico colombiano (septiembre-octubre 2004). Investigaciones Marinas, Valparaíso, 34: 211-216.

Rodríguez-Rubio, E. \& Stuardo, J. (2002). Variability of photosynthetic pigments in the Colombian Pacific Ocean and its relationship with the wind field using ADEOS-I data. Proceedings of the Indian Academy of Science (Earth and Planetary Science Letters), 111: 1-10.

Rodríguez-Rubio, E., Schneider, W. \& Abarca del Rio, R. (2003). On the seasonal circulation within the Panama Bight derived from satellite observations of wind, altimetry and sea surface temperature. Geophysical Research Letters, 30: 1410-1413.
Rubao, J., Edwards, M., Mackas, D. L., Runge, J. A. \& Thomas, A. C. (2010). Marine plankton phenology and life history in a changing climate: current research and future directions. Journal of Plankton Research, 32(10): 1355-1368.

Smayda, T. J. (1966). A quantitative analysis of the phytoplankton of the Gulf of Panama: III. general ecological conditions, and the phytoplankton dynamics at $8^{\circ} 45^{\prime} \mathrm{N}, 79^{\circ} 23^{\prime} \mathrm{W}$ from November 1954 to Mau 1957. Inter-American Tropical Tuna Commission, 11: 355-612.

Soto, P. A., Sánchez, S. L. \& Fernández, C. (2001). Comunidades planctónicas marinas. In L. M. Barrios \& M. L. Victoria (Eds.), Gorgona marina: Contribución al conocimiento de una isla única (pp. 93-105.). INVEMAR, Serie de Publicaciones especiales $N^{\circ}$ 7. Santa Marta, Colombia.

StatSoft, Inc. 2004. STATISTICA (data analysis software system), version 7 .

Tomas, C. (1993). Marine phytoplankton: a guide to naked flagellates and coccolithophorids. Academica Press, San Diego.

Tomas , C. (1997). Identifying marine phytoplankton. Academia Press. San Diego.

Valencia, B. \& Giraldo, A. (2012). Structure of hyperiid amphipod assemblages on Isla Gorgona, eastern tropical Pacific off Colombia. Journal of the Marine Biological Association of the United Kingdom, 92(7): 1489-1499. 